\title{
Group Selection May Explain Cancer Predisposition and Other Human Traits' Evolution
}

\author{
Konstantinos Voskarides ${ }^{1}$
}

Received: 16 March 2018 / Accepted: 3 April 2018 / Published online: 5 April 2018

○) Springer Science+Business Media, LLC, part of Springer Nature 2018

\begin{abstract}
Group selection is a matter of acute controversy among evolutionary biologists. The most well-publicized debate in this regard is that between Edward O. Wilson and Richard Dawkins. As is widely known, Edward O. Wilson is very excited about the idea of social selection and eusociality; by contrast Richard Dawkins favors the idea of gene selection. As is often the case, the truth is somewhere in the middle. Evolution is most likely a multilevel procedure, where selection forces act on genes, individuals, and groups. Here, I would like to emphasize that group selection may be a possible cause of increased genetic variation on DNA repair genes, subsequently this driving to high cancer incidence. Additionally, if group selection is indeed happening in humans, maybe this is the reason that few adaptive loci have been discovered in human genome, even though thousands of sequenced genomes exist today.
\end{abstract}

Keywords Natural selection $\cdot$ DNA repair genes $\cdot$ Mismatch repair $\cdot$ Extreme environments · Adaptation · Group selection

Group selection is a matter of acute controversy among evolutionary biologists. The most well-publicized debate in this regard is that between Edward O. Wilson and Richard Dawkins. As is widely known, Edward O. Wilson is very excited about the idea of social selection and eusociality; by contrast Richard Dawkins favors the idea of gene selection. As is often the case, the truth is somewhere in the middle. Evolution is most likely a multilevel procedure, where selection forces act on genes, individuals, and groups (Pievani 2014). Here, I would like to emphasize that group selection may be a possible cause of increased genetic variation on DNA repair genes, subsequently this driving to high cancer incidence. Additionally, if group selection is indeed happening in humans, maybe this is the reason that few adaptive loci have been discovered in human genome, even though thousands of sequenced genomes exist today.

Anthropological and archeological studies have uncovered a lot of evidence that during Paleolithic and early Neolithic eras humans used to live in small groups of individuals. Such small groups can be evolutionarily anticipated to behave as though they were single individuals (Pievani

Konstantinos Voskarides

kvoskar@ucy.ac.cy

1 Medical School, University of Cyprus, Nicosia, Cyprus
2014). Highly adaptive groups survived, while the others have gone extinct. This procedure was happening for thousands of years as humans experienced very difficult environmental conditions such as extreme cold and lack of food. I propose that this is a possible reason for elucidation of very few adaptive genetic loci in the human genome today (cf. Grossman et al. 2013). In my mind, current evolutionary genetics methods that use mainly linkage disequilibrium and genetic polymorphisms frequencies are inadequate to detect the previous action of group selection in humans. Groups that have survived transmitted to their descendants a "package" of phenotypic traits, leaving little evidence for previous selective contributions. Additionally, as I will explain below, those evolutionarily successful groups of individuals were probably very prone to mutagenesis.

In a recent article of Thomas et al. (2016), an attractive explanation is proposed as to why cancer incidence is much higher in specific human organs, when compared with other organs. Their theoretical approach proposes that natural selection has shaped increased protection against cancer for small, essential, and unpaired organs compared with larger, less essential, and often paired organs. They give substantial examples to support this hypothesis, e.g., the fact that heart and brain cancers are very rare, because these organs are of viable importance for survival, in contrast with cancers in other organs like the colon and 
breasts. Despite this, I believe that there is an equally satisfactory evolutionary explanation for the high incidence of these cancers. This explanation is based on the fact that our ancestors had experienced group selection at the past, due to long exposure to extreme environmental conditions, and probably this was a cause for adaption of "cancerous" genetic variability in DNA repair genes. Cancer may be a "side-effect" of evolution.

The main point of this theory is that mutations that disturb the normal function of DNA repair genes can give the opportunity to a population to increase its mutation (genetic) load, this subsequently increasing the available phenotypic variability. This can often make the difference between survival and extinction of a species. When environment suddenly changes, and when climatic conditions become extreme, then group selection becomes potentiated. Groups with higher mutation load, the ones that happened to have more mutations on DNA repair genes, have better chance to adapt in the new environment (Fig. 1). The problem is that when environment returns to "normal," selection cannot discard these mutations (on DNA repair genes) out of the population. Improved environmental conditions or technological advances can increase life expectancy. The result is an increased rate of cancers (Fig. 1) that are closely related with mutations on DNA repair genes (Li and Martin 2016; Kleibl and Kristensen 2016). Most of these cancers present at old and reproductively inactive ages, when selection is too weak to be effective.

Breast cancer and colorectal cancer are among the most frequent cancers today, especially in developed and developing countries. Additionally, these two cancer types are the most known to be closely related with DNA repair genes mutations or genetic polymorphisms. This is an indication that human genome has accumulated a lot of "cancerous" genetic variation on DNA repair genes. Unfortunately, this "cancerous" genetic variation is being expressed today due to the increase of human life expectancy. Recent findings give evidence that mutations that helped humans to survive at extreme cold and at extreme high altitude also appear to contribute to increased cancer incidence today (Voskarides 2018).

Group selection is a well-documented phenomenon in single-celled organisms. "Mutator" strains are bacterial populations with increased capability of introducing new mutations. Such strains appear suddenly at bacterial cultures, when these cultures grow under stressful conditions, e.g., lack of food (Cox et al. 1972). Bacterial cultures are grown from multiple colonies. Each colony is a "group of individuals." Colonies with adaptive characteristics survive and gradually displace the others. Mutator strains were also discovered in yeast cultures growing under stressful conditions (Bui et al. 2015). The most interesting point here
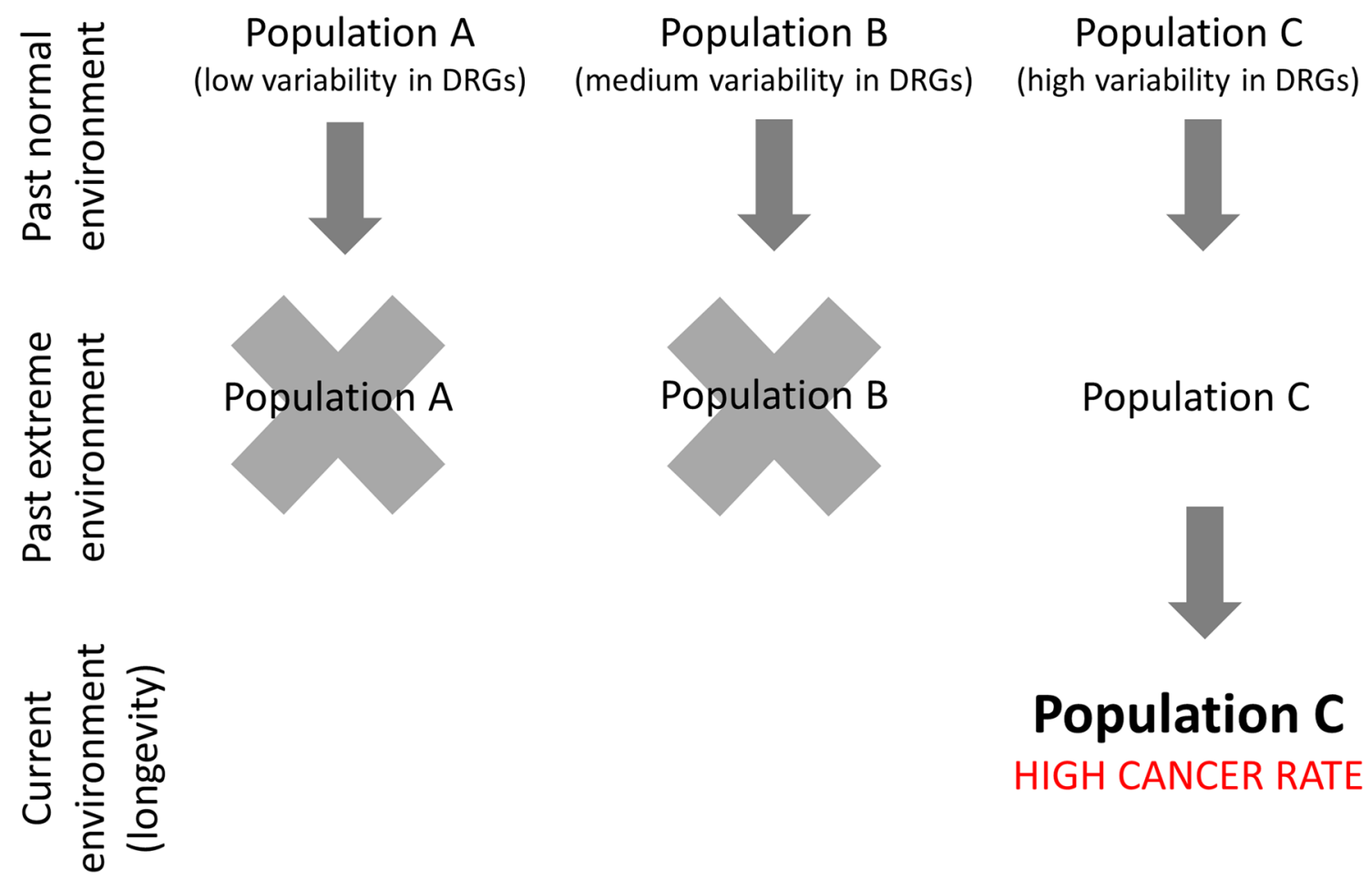

\section{Population C}

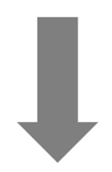

\section{Population C HIGH CANCER RATE}

Fig. 1 Group selection that our species may have experienced under extreme environmental conditions. Human sub-populations with increased genetic variability in DNA repair genes (DRGs) had a better chance to survive. We are the progenies of these populations (Population C). Unfortunately, due to longevity, genetic variation that contributed to our survival it is presently a cause of high cancer rate 
is that these mutator strains have mutations in mismatch DNA repair genes, the same genes that, when mutated, cause or predispose for colon cancer in humans (e.g., the Msh2 gene). No experimental molecular studies have been performed so far for the existence of "mutators" in multicellular organisms.

Concluding, I would like to emphasize that evolutionary theories, such as that for group selection, help us to reexamine the phenomenon of cancer and probably other diseases under a new conceptual framework. This concept could redefine our therapeutic strategies. We must not forget that the evolutionary theory of micro-environment of cancer cells ultimately determined the strategy of anti-cancer combinational therapies (Gillies et al. 2012; Enriquez-Navas et al. 2015). Group selection is an idea that needs revisiting and experimental examination.

\section{References}

Bui DT, Dine E, Anderson JB, Aquadro CF, Alani EE (2015) A genetic incompatibility accelerates adaptation in yeast. PLoS Genet 11(7):e1005407
Cox EC, Degnen GE, Scheppe ML (1972) Mutator gene studies in Escherichia coli: the mutS gene. Genetics 72(4):551-567

Enriquez-Navas PM, Wojtkowiak JW, Gatenby RA (2015) Application of evolutionary principles to cancer therapy. Cancer Res 75(22):4675-4680

Gillies RJ, Verduzco D, Gatenby RA (2012) Evolutionary dynamics of carcinogenesis and why targeted therapy does not work. Nat Rev Cancer 12(7):487-493

Grossman SR, Andersen KG, Shlyakhter I, Tabrizi S, Winnicki S, Yen A, Park DJ, Griesemer D, Karlsson EK, Wong SH, Cabili M, Adegbola RA, Bamezai RN, Hill AV, Vannberg FO, Rinn JL, 1000 Genomes Project, Lander ES, Schaffner SF, Sabeti PC (2013) Identifying recent adaptations in large-scale genomic data. Cell 152(4):703-713

Kleibl Z, Kristensen VN (2016) Women at high risk of breast cancer: molecular characteristics, clinical presentation and management. Breast 28:136-144

Li SK, Martin A (2016) Mismatch repair and colon cancer: mechanisms and therapies explored. Trends Mol Med 22(4):274-289

Pievani T (2014) Individuals and groups in evolution: Darwinian pluralism and the multilevel selection debate. J Biosci 39(2):319-325

Thomas F, Nesse RM, Gatenby R, Gidoin C, Renaud F, Roche B, Ujvari B (2016) Evolutionary ecology of organs: a missing link in cancer development? Trends Cancer 2(8):409-415

Voskarides K (2018) Combination of 247 genome-wide association studies reveals high cancer risk as a result of evolutionary adaptation. Mol Biol Evol 35(2):473-485 